\title{
НЕЛІНІЙНІ ПЛАЗМОВІ ДИПОЛЬНІ КОЛИВАННЯ У СФЕРОЇДАЛЬНИХ МЕТАЛЕВИХ НАНОЧАСТИНКАХ
}

\author{
П.М. ТОМЧУК, Д.В. БУТЕНКО
}

УдК 538.94, 537.5

Інститут фізики НАН України, відділ теоретичної фізики

(C) 2011

(Просn. Науки, 46, Kü̈в 03680; e-mail: ptomchuk@iop. kiev. ua)

$\mathrm{У}$ роботі розвинуто теорію нелінійних дипольних плазмових коливань у металевій наночастинці сфероїдальної форми, які генеруються полем лазерної хвилі. Для випадку лазерного поля, орієнтованого вздовж осі обертання сфероїда, отримано наближені аналітичні вирази для дипольного моменту наночастинки (з точністю до кубічної складової).

\section{1. Вступ}

При зміщенні положення центра мас електронної підсистеми металевої наночастинки відносно положення центра мас іонної підсистеми виникає електростатична сила, яка протидіє просторовому рознесенню згаданих центрів мас. 3 наявністю такої сили пов'язано виникнення в металевих наночастинках дипольних плазмових коливань. Ця сила в першому наближенні пропорційна взаємному зміщенню електронного і іонного центрів мас. Під час збільшення величини зміщення електростатична сила починає нелінійно залежати від цього зміщення, що приводить до появи нелінійних дипольних плазмових коливань. Такі нелінійні плазмові коливання в металевих наночастинках вивчено в роботах [1-3]. У роботах $[1,2]$ плазмові коливання розглядали в континуальному наближенні, а в [3] за основу було взято мікроскопічний підхід. В усіх названих роботах форму металевої наночастинки прийнято сферичною.

Нашу роботу присвячено теорії нелінійних плазмових коливань в металевих наночастинках еліпсоїдальної форми. Варто підкреслити, що результати теорії плазмових резонансів в асиметричних металевих наночастинках не зводяться до малих поправок до відомих результатів для сферичних частинок, а мають принципові відмінності. Так, вже у лінійному наближенні сферично симетрична металева частинка має один плазмовий резонанс, частинка сфероїдальної форми - два, а еліпсоїдальної - три. Тому задача побудови нелінійної теорії дипольних плазмових коливань в асиметричних металевих наночастинках залишається на сьогодні актуальною і цікавою.

\section{2. Постановка задачі}

Задачу про коливні процеси в металевих наночастинках будемо розглядати в континуальному наближенні, взявши за основу (подібно до [2]) гідродинамічні рівняння для електронної густини $n_{e}(\mathbf{r}, t)$ і електронної швидкості $\boldsymbol{v}(\mathbf{r}, t)$ :

$\frac{\partial n_{e}}{\partial t}+\nabla\left(n_{e} \boldsymbol{v}\right)=0$

$\frac{\partial \boldsymbol{v}}{\partial t}+(\boldsymbol{v} \boldsymbol{\nabla}) \boldsymbol{v}=\frac{\mathbf{F}}{m_{e}} \equiv \frac{1}{m_{e}} \times$

$\times\left\{-e \mathbf{E}_{L}+e\left[\nabla \Phi_{e}+\nabla \Phi_{i}\right]-\frac{\nabla p}{n_{e}}\right\}$,

де $\mathbf{F}(\mathbf{r}, t)$ - повна сила, яка діє на електронну рідину. Вона складається 3 дії електричного поля лазерної хвилі $\left(\mathbf{E}_{L}\right)$, а також дії градієнтів електронного $\left(\Phi_{e}\right)$ та іонного $\left(\Phi_{i}\right)$ потенціалів і градієнта тиску $(p)$.

Поле $\mathbf{E}_{L}$ в межах наночастинки в дипольному наближенні вважається просторово однорідним.

Введемо вектор, який характеризує положення центра мас електронної підсистеми:

$\mathbf{u}(t)=N_{e}^{-1} \int d^{3} r n_{e}(\mathbf{r}, t) \mathbf{r}$ 
де $(3) N_{e}$ - повна кількість електронів у металевій наночастинці.

Рівняння руху для центра мас електронної підсистеми з використанням (1) зводимо до вигляду

$$
\begin{aligned}
& N_{e} \frac{d^{2} \mathbf{u}}{d t^{2}}=\int d^{3} r \frac{\partial^{2} n_{e}(\mathbf{r}, t)}{\partial t^{2}} \mathbf{r}= \\
& =\int d^{3} r n_{e}(\mathbf{r}, t)\left(\frac{\partial \boldsymbol{v}}{\partial t}+(\boldsymbol{v} \nabla) \boldsymbol{v}\right) .
\end{aligned}
$$

Якщо це рівняння записати у формі

$m_{e} \mathbf{u}=N_{e}^{-1}\langle\mathbf{F}\rangle$,

то із (4) і (2) отримуємо

$\langle\mathbf{F}\rangle=-e E_{L}(t) N_{e}+$

$+\int d^{3} r\left\{e\left[\nabla \Phi_{e}(\mathbf{r}, t)+\nabla \Phi_{i}(\mathbf{r}, t)\right] n_{e}(\mathbf{r}, t)-\nabla p(\mathbf{r}, t)\right\}$

Вище ми коротко повторили підхід до плазмових дипольних коливань у металевих наночастинках, прийнятий у роботі [2]. Далі у [2] після необхідних оцінок прийнято наближення, суть якого зводиться до припущення, що електронна підсистема наночастинки як єдине ціле (без деформацій) зміщується разом зі зміщенням електронного центра мас. Це означає, що можна покласти:

$n_{e}(\mathbf{r}, t)=n_{e}^{(0)}(|\mathbf{r}-\mathbf{u}(t)|)$,

$\Phi_{e}(\mathbf{r}, t)=\Phi_{e}^{(0)}(|\mathbf{r}-\mathbf{u}(t)|)$,

$\mathbf{p}(\mathbf{r}, t)=p_{0}(|\mathbf{r}-\mathbf{u}(t)|)$.

Враховуючи те, що при термодинамічній рівновазі (за відсутності $\left.\mathbf{E}_{L}(t)\right)$ має місце рівність

$e\left[\nabla \Phi_{e}^{(0)}+\nabla \Phi_{i}^{(0)}\right]-\frac{\nabla \rho^{(0)}}{n_{e}}=0$

iз (6) i (7) отримуємо

$$
\begin{aligned}
& \langle\mathbf{F}\rangle=-e \mathbf{E}_{L}(t) N_{e}+\int d^{3} r e \nabla \Phi_{i}^{(0)}(\mathbf{r}) n_{e}^{(0)}(|\mathbf{r}-\mathbf{u}(t)|)= \\
& =-e \mathbf{E}_{L}(t) N_{e}+\frac{\partial}{\partial \mathbf{u}} \int d^{3} r e \Phi_{i}^{(0)}(\mathbf{r}) n_{e}^{(0)}(|\mathbf{r}-\mathbf{u}|)
\end{aligned}
$$

Ця формула буде для нас вихідною.

Далі на відміну від попередніх робіт [1-3], в яких плазмові нелінійні коливання розглядались у сферично симетричних металевих наночастинках, будемо мати справу з асиметричними наночастинками. Будемо вважати, що металева наночастинка має форму еліпсоїда обертання (сфероїда). Спрямуємо вісь координат $0 Z$ вздовж осі симетрії сфероїда. Крім того, будемо допускати, що лазерне поле $\mathbf{E}_{L}(t)$ і викликане ним зміщення центра мас електронної підсистеми спрямовані вздовж осі $0 Z$. Щоб підкреслити цей факт, далі запишемо так:

$\mathbf{u} \equiv \mathbf{z}_{0}$

Зауважимо, що коли форма частинки відрізняється від сферичної і генеруюче плазмові коливання лазерне поле не орієнтоване строго вздовж осі симетрії, то в нелінійному наближенні різні плазмові резонанси зав'язані між собою, і задача неймовірно ускладнюється. Як показують подальші викладки вже наведена вище найпростіша модель відхилення від сферичної симетрії дає якісно нові результати порівняно зі сферичним випадком.

Досі, за винятком формул (7) і (9), ніде симетрія наночастинки не конкретизувалась. Ми так само, як і в [2], будемо допускати, що електронна підсистема як єдине ціле (без диформацій) зміщується разом із центром мас.

Отже, приймемо

$n_{e}(\mathbf{r}, t)=n_{0} \Delta_{0}\left(R(\theta)-\left|\mathbf{r}-\mathbf{z}_{0}\right|\right)$,

де (11) $n_{0}$ - концентрація електронів, $\Delta_{0}(x)$ - сходинкоподібна функція

$\Delta_{0}(x)= \begin{cases}1, & x>0 \\ 0, & x<0\end{cases}$

Крім того, $R(\theta)$ в (11) задає поверхню сфероїда:

$R(\theta)=\frac{R_{\perp}}{\sqrt{1-e_{p}^{2} \cos ^{2} \theta}}$.

Для конкретизації ми розглядаємо витягнутий сфероїд $e_{p}$ - ексцентриситет, $\theta-$ кут між віссю $0 Z$ і вектором $\mathbf{R}(\theta)$ на поверхні сфероїда:

$e_{p}^{2}=\left|\frac{R_{\perp}^{2}}{R_{\|}^{2}}-1\right|$,

$R_{\|}$- поздовжній (вздовж осі симетрії) i $R_{\perp}$ - поперечний радіус кривизни. 
Якщо тепер аналогічно (7) допускати, що $\Phi_{e}(\mathbf{r}, t)$ i $p(\mathbf{r}, t)$ подібна до структури $n_{e}(\mathbf{r}, t)$, заданої формулою (11), то ми замість (9) отримуємо

$$
\begin{aligned}
& \langle\mathbf{F}\rangle=-e \mathbf{E}_{L}(t) N_{e}+n_{0} \times \\
& \times \int d^{3} r e \bar{\nabla} \Phi_{i}^{(0)}(\mathbf{r}) \Delta_{0}\left(R(\theta)-\left|\mathbf{r}-\mathbf{z}_{0}\right|\right) .
\end{aligned}
$$

Отже, щоб визначити силу $\langle\mathbf{F}\rangle$, яка протидіє зміщенню вздовж осі $0 Z$ електронної підсистеми сфероїдальної металевої наночастинки, нам потрібно знайти вигляд іонного електростатичного потенціалу $\Phi_{i}^{(0)}$. Це ми будемо робити в наступному розділі.

\section{3. Електростатичний потенціал зарядженого сфероїда}

Електростатичний потенціал іонного остова сфероїдальної наночастинки має вигляд

$\Phi_{i}^{(0)}=\int_{V} \frac{\rho_{i} d^{3} r}{\left|\mathbf{r}-\mathbf{r}^{\prime}\right|}$

Вважаємо, що густина іонного заряду рівномірно розподілена по об'єму $V$ :

$\rho_{i}=e N_{i} Z_{i} / V=$ const,

де $N_{i}$ - кількість іонів, $Z_{i}-$ кратність заряду.

Враховуючи те, що маємо справу зі сфероїдом і що густина $\rho_{i}$ - стала, розпишемо (16) так:

$\Phi_{i}^{(0)}=\rho_{i} \int_{0}^{2 \pi} d \varphi^{\prime} \int_{0}^{\pi} d \theta^{\prime} \sin \theta^{\prime} \int_{0}^{R\left(\theta^{\prime}\right)} \frac{d r^{\prime} r^{\prime 2}}{\left|\mathbf{r}-\mathbf{r}^{\prime}\right|}$.

Далі, щоб взяти інтеграл (18), використаємо розклад:

$\frac{1}{\left|\mathbf{r}-\mathbf{r}^{\prime}\right|}=\sum_{n=0}^{\infty} P_{n}(\cos \nu)\left\{\begin{array}{lll}\frac{1}{r}\left(\frac{r^{\prime}}{r}\right)^{n} & \text { at } & r^{\prime}<r, \\ \frac{1}{r^{\prime}}\left(\frac{r}{r^{\prime}}\right)^{n} & \text { at } & r^{\prime}>r,\end{array}\right.$

де $\nu$-кут між вектором $\mathbf{r} i \mathbf{r}^{\prime}$.

Тепер використаємо відоме співвідношення [4]:

$P_{n}(\cos \nu)=P_{n}\left(\cos \theta^{\prime}\right) P_{n}(\cos \theta)+$

$+2 \sum_{m} \frac{(n-m) !}{(n+m) !} \cos \left(\varphi^{\prime}-\varphi\right) P_{n}^{m}\left(\cos \theta^{\prime}\right) P_{n}^{m}(\cos \theta)$.

У $(20)$ кути $\left(\varphi^{\prime}, \theta^{\prime}\right)$ і $(\varphi, \theta)$ задають, відповідно, орієнтацію векторів $\mathbf{r}^{\prime}$ і $\mathbf{r}$.
При підстановці (20) в (18) другий член в (20) зануляється при інтеграції по $\varphi^{\prime}$.

Оскільки $R\left(\theta^{\prime}\right)$ у (18) згідно з (13) задовольняє умову

$R_{\perp} \leq R\left(\theta^{\prime}\right) \leq R_{\|}$

то доцільно розбити інтегрування на три випадки:

a) $r \leq R_{\perp}$

b) $R_{\perp} \leq r \leq R_{\|}$

c) $R_{\|} \leq r$.

a) при $r \leq R_{\perp}$ згідно з (18), (19) і (20) отримуємо

$\Phi_{i}^{(0)}=2 \pi \rho_{i} \sum_{n=0}^{\infty} P_{n}(\cos \theta) \int_{0}^{\pi} d \theta^{\prime} \sin \theta^{\prime} P_{n}\left(\cos \theta^{\prime}\right) \times$

$\times\left\{\frac{1}{r} \int_{0}^{r} d r^{\prime} r^{\prime 2}\left(\frac{r^{\prime}}{r}\right)^{n}+\int_{r}^{R\left(\theta^{\prime}\right)} d r^{\prime} r^{\prime}\left(\frac{r}{r^{\prime}}\right)^{n}\right\}$.

Подальше обчислення інтегралів у (23) труднощів не має. Більш складна ситуація виникає у випадку $b$.

b) $R_{\perp} \leq r \leq R_{\|}$, оскільки згідно з (21) у цьому ж діапазоні змінюється також $r^{\prime}$, то залежно від кута $\theta^{\prime}$ максимальне значення $r^{\prime}$ може бути як більше, так і менше за $r$ (див. рис. 1). Доцільно ввести кут $\theta_{1}$, за якого еліпсоїд і сфера радіуса $r$ перетинаються, тобто

$R\left(\theta^{\prime}\right)=R\left(\theta_{1}\right)=r$.

Із (24) з урахуванням (13) отримуємо

$\cos \theta_{1}=\frac{1}{e_{\rho}}\left\{1-\left(\frac{R_{\perp}^{2}}{r}\right)\right\}^{1 / 2}$

Тепер інтеграл по $\theta^{\prime}$ в (18) розіб'ємо так:

$\int_{0}^{\pi} d \theta^{\prime} \ldots=\int_{0}^{\theta_{1}} d \theta^{\prime} \ldots+\int_{\theta_{1}}^{\pi-\theta_{1}} d \theta^{\prime} \ldots+\int_{\pi-\theta_{1}}^{\pi} d \theta^{\prime} \ldots$

В інтервалах $\left(0 \rightarrow \theta_{1}\right)$ і $\left(\pi-\theta_{1} \rightarrow \pi\right)$ величина $r^{\prime}$, як видно з рисунка, може бути як більшою, так і меншою за $r$, а в інтервалі: $\left(\theta_{1} \rightarrow \pi-\theta_{1}\right) r^{\prime}$ завжди менша за $r$. 


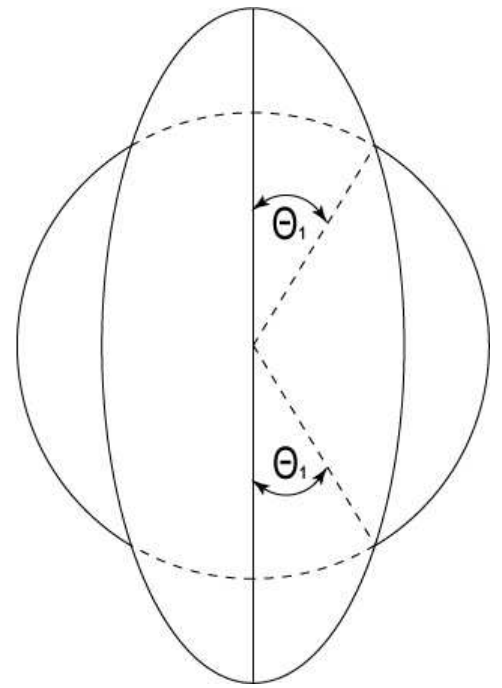

Рис. 1

Підставимо тепер розклад (19) у (18) і використаємо розбиття інтеграції по куту $\theta^{\prime}$ відповідно до (26), отримуємо

$\Phi_{i}^{(0)}=2 \pi \rho_{i} \sum_{n=0}^{\infty} P_{n}(\cos \theta)\left\{\int_{0}^{\theta_{1}} d \theta^{\prime} \sin \theta^{\prime} P_{n}\left(\cos \theta^{\prime}\right) \times\right.$

$\times\left[\frac{1}{r} \int_{0}^{r} d r^{\prime} r^{\prime 2}\left(\frac{r^{\prime}}{r}\right)^{n}+\int_{r}^{R\left(\theta^{\prime}\right)} d r^{\prime} r^{\prime}\left(\frac{r}{r^{\prime}}\right)^{n}\right]+$

$+\int_{\theta_{1}}^{\pi-\theta_{1}} d \theta^{\prime} \sin \theta^{\prime} P_{n}\left(\cos \theta^{\prime}\right) \frac{1}{r} \int_{0}^{R\left(\theta^{\prime}\right)} d r^{\prime} r^{\prime 2}\left(\frac{r^{\prime}}{r}\right)^{n}+$

$+\int_{\pi-\theta_{1}}^{\pi} d \theta^{\prime} \sin \theta^{\prime} P_{n}\left(\cos \theta^{\prime}\right) \times$

$\left.\times\left[\frac{1}{r} \int_{0}^{r} d r^{\prime} r^{\prime 2}\left(\frac{r^{\prime}}{r}\right)^{n}+\int_{r}^{R\left(\theta^{\prime}\right)} d r^{\prime} r^{\prime}\left(\frac{r}{r^{\prime}}\right)^{n}\right]\right\}$.

Замінивши в останньому члені змінні (типу $\theta^{\prime} \rightarrow \pi-$ $\left.\theta^{\prime}\right)$ в останньому члені (27), виразу (27) можна надати вигляду

$\Phi_{i}^{(0)}=2 \pi \rho_{i} \sum_{n=0}^{\infty} P_{n}(\cos \theta)\left\{\int_{0}^{\theta_{1}} d \theta^{\prime} \sin \theta^{\prime} \times\right.$
$\times\left[P_{n}\left(\cos \theta^{\prime}\right)+P_{n}\left(-\cos \theta^{\prime}\right)\right] \times$

$\times\left[\frac{1}{r} \int_{0}^{r} d r^{\prime} r^{\prime 2}\left(\frac{r^{\prime}}{r}\right)^{n}+\int_{r}^{R\left(\theta^{\prime}\right)} d r^{\prime} r^{\prime}\left(\frac{r}{r^{\prime}}\right)^{n}\right]+$

$+\int_{\theta_{1}}^{\pi / 2_{1}} d \theta^{\prime} \sin \theta^{\prime}\left[P_{n}\left(\cos \theta^{\prime}\right)+P_{n}\left(-\cos \theta^{\prime}\right)\right] \times$

$\left.\times \frac{1}{r} \int_{0}^{R\left(\theta^{\prime}\right)} d r^{\prime} r^{\prime 2}\left(\frac{r^{\prime}}{r}\right)^{n}\right\}$

3 (28) видно, що всі непарні по $n$ члени в сумі (28) випадають. Вираз (28) дає вигляд $\Phi_{i}^{(0)}$ в діапазоні $R_{\perp} \leq r \leq R_{\|}$

Нарешті розглянемо випадок

c) $r \geq R_{\|}$. У цьому випадку $r^{\prime}<r$. Із (18) отримуємо

$\Phi_{i}^{(0)}=2 \pi, \rho_{i} \sum_{n=0}^{\infty} P_{n}(\cos \theta) \int_{0}^{\pi} d \theta^{\prime} \sin \theta^{\prime} \times P_{n}\left(\cos \theta^{\prime}\right) \times$

$\times \frac{1}{r} \int_{0}^{R\left(\theta^{\prime}\right)} d r^{\prime} r^{\prime 2}\left(\frac{r^{\prime}}{r}\right)^{n}$

Для всього діапазону зміни $\mathbf{r}$, як бачимо із виразів (23), (28) і (29), можемо записати

$\Phi_{i}^{(0)}=\sum_{n=0}^{\infty} P_{n}(\cos \theta) \Psi(n)=\Psi(0)+$

$+P_{2}(\cos \theta) \Psi(2)+P_{4}(\cos \theta) \Psi(4)+\cdots$.

Взявши інтеграли в (23), (28) і (29), отримаємо вирази для коефіцієнтів $\Psi(n)$. Зокрема при $r \leq R_{\perp}$ із (23) знаходимо

$\Psi(0)=2 \pi \rho_{i}\left\{-\frac{r^{2}}{3}+\frac{R_{\perp}^{2}}{2 e_{p}} \ln \left(\frac{1+e_{p}}{1-e_{p}}\right)\right\} ; r \leq R_{\perp}$.

При $R_{\perp} \leq r \leq R_{\|}$із (28) отримуємо

$\Psi(0)=2 \pi \rho_{i}\left\{\frac{r^{2}}{3}\left(\cos \theta_{1}-1\right)-\right.$

ISSN 2071-0194. Укр. фіз. журн. 2011. T. 56, №10

\section{4}


$\left.-\frac{R_{\perp}^{2}}{2 e_{p}} \ln \left(\frac{1+e_{p} \cos \theta_{1}}{1-e_{p} \cos \theta_{1}} \frac{1-e_{p}}{1+e_{p}}\right)+\frac{2 R_{\perp}^{2}}{3} \cos \theta_{1}\right\}$

$R_{\perp} \leq r \leq R_{\|}$

Зрештою, при $r \geq R_{\|}$із (29) будемо мати:

$\Psi(0)=\frac{4}{3} \pi \rho_{i} R_{\perp}^{2} R_{\|} R_{\|} / r, \quad r \geq R_{\|}$.

Аналогічні вирази для $\Psi(2)$ наведено в додатку.

Як видно із (25), при $r=R_{\perp}$ маємо $\cos \theta_{1}=0$, і тоді (32) збігається з (31), а при $r=R_{\|}$буде $\cos \theta_{1}=1$, i тоді вираз (32) збігається з виразом (33).

Якщо зробити граничний перехід від еліпсоїдальної форми до сферичної, тобто $e_{p} \rightarrow 0$ (при цьому $\left.R_{\perp}=R_{\|} \equiv R\right)$, то легко бачити, що при $n \neq 0$ всі $\Psi(n) \rightarrow 0$. Що стосується $\Psi(0)$, то за такого переходу, як видно із (31)-(33), будемо мати

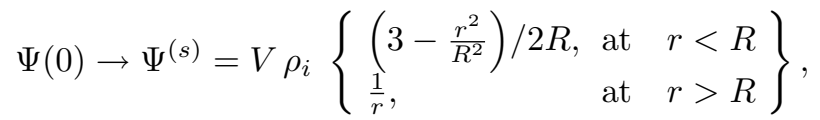

де $V=\frac{4 \pi}{3} R^{3}$ - об'єм сфери. Вираз для $\Psi^{(s)}$ у формі (34) було використано в [2] під час розгляду нелінійних плазмових коливань у сферичній металевій наночастинці.

\section{4. Електростатична сила}

При орієнтації поля $\mathbf{E}_{L}(t)$ вздовж осі $0 Z$ і прийнятій нами симетрії форми частинки поле $\langle F\rangle$ буде також спрямоване вздовж $0 Z$, тобто

$\langle F\rangle=-e E_{L}(t) N_{e}+$

$+n_{0} e \int d^{3} r \mathbf{K}_{0} \nabla \Phi_{i}^{(0)}(\mathbf{r}) \Delta_{0}\left(R(\theta)-\left|\mathbf{r}-\mathbf{z}_{0}\right|\right)$,

де $\mathbf{K}_{0}$ - орт вздовж осі $0 Z$.

Область інтегрування по $\mathbf{r}$ в (35) визначається 3 умови, що аргумент у сходинкоподібній функції $\Delta_{0}$ більший або дорівнює нулеві, тобто

$R(\theta)-\left|\mathbf{r}-\mathbf{z}_{0}\right| \geq 0$

З умови рівності в (36) отримуємо корінь

$r \equiv r_{m}(\nu)=\left\{R^{2}(\theta)-z_{0}^{2} \sin ^{2} \nu\right\}^{1 / 2}+z_{0} \cos \nu$.

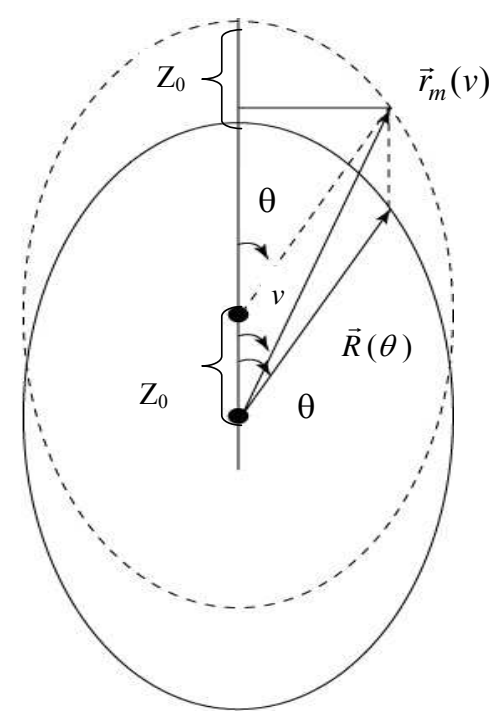

Рис. 2

Як видно з рис. 2, вектор $\mathbf{r}_{m}(\nu)$ задає на поверхні зсунутого сфероїда положення тієї точки, яка на поверхні первісного сфероїда задавалась вектором $\mathbf{R}(\theta)$. Вектор $\mathbf{R}(\theta)$ при зміщенні переноситься паралельно собі із точки 0 в точку $0^{\prime}$. Як видно з рис. 2 , можемо записати

$\mathbf{r}_{m}(\nu) \cos \nu=z_{0}+R(\theta) \cos \theta$

$\mathbf{r}_{m}(\nu) \sin \nu=R(\theta) \sin \theta$.

Якщо домножити співвідношення (38) на $\sin \nu$, а (39) на $\cos \nu$ і відняти один від одного, то отримуємо

$z_{0} \sin \nu=R(\theta) \sin (\theta-\nu)$.

Ця формула при заданому $z_{0}$ встановлює зв'язок кутів $\theta i \nu$. При $z \rightarrow 0$ маємо $\theta \rightarrow \nu$. Оскільки $z_{0} / R(\nu)$ мала величина, то можна записати

$\theta=\nu+\Delta \nu$

і визначити величину $\Delta \nu$ iз (40) рекурентним способом:

$\Delta \nu_{1}=\frac{z_{0}}{R(\nu)} \sin \nu$

$\Delta \nu_{2}=\frac{z_{0}}{R(\nu)} \sin \nu\left(1-\frac{R^{\prime}(\nu)}{R(\nu)} \Delta \nu_{1}\right)$

$\Delta \nu_{3}=\frac{z_{0}}{R(\nu)} \sin \nu\left(1-\frac{R^{\prime}(\nu)}{R(\nu)} \Delta \nu_{2}\right), \ldots$ 
Щоб отримати явну залежність $\mathbf{r}_{m}(\nu)$ від зміщення $z_{0}$ розкладемо (37) у ряд (розкладемо також $R(\theta)$ з використанням співвідношень (41), (42)):

$r_{m}(\nu)=R(\nu)+z_{0}\left(1-e_{p}^{2}\right) \frac{\cos \nu}{1-e_{p}^{2} \cos ^{2} \nu}-$

$-z_{0}^{2} \frac{1-e_{p}^{2}}{2 R_{\perp}} \frac{\sin ^{2} \nu}{\left(1-e_{p}^{2} \cos ^{2} \nu\right)^{3 / 2}}+$

$+z_{0}^{3} \frac{e_{p}^{2}}{2 R_{\perp}^{2}}\left(\frac{1}{3}+\frac{1-e_{p}^{2}}{\left(1-e_{p}^{2} \cos ^{2} \nu\right)^{2}}\right) \cos \nu \sin ^{4} \nu+\ldots$

Для випадку сферичної форми $\left(e_{p} \rightarrow 0\right)$ із (37) знаходимо

$\mathbf{r}_{m}(\nu)=R+z_{0} \cos \nu-\frac{z_{0}^{2}}{2 R} \sin ^{2} \nu-\frac{z_{0}^{4}}{8 R^{3}} \sin ^{4} \nu+\ldots$

Порівнюючи (43) і (44), бачимо, що в асиметричній частинці $\left(e_{p} \neq 0\right)$ додатково з'являється кубічна нелінійність, яка відсутня в (44).

Щоб уникнути надалі непорозумінь наголосимо, що під терміном "асиметрична частинка" ми розуміємо частинку, форма якої відрізняється від сферичної (а не відсутність у частинці будь-яких елементів симетрії).

Зауважимо, що формулу (35) з урахуванням (36), (37) можна звести до вигляду

$\langle F\rangle=-e E_{L}(t) N_{e}+$

$+2 \pi n_{0} e \int_{0}^{\pi} d \nu \sin \nu \int_{0}^{r_{m}(\nu)} d r r^{2}\left[\mathbf{K}_{0} \nabla \Phi_{i}^{(0)}(r)\right]$.

Якщо записати $r_{m}(\nu)$ у вигляді

$\mathbf{r}_{m}(\nu)=R(\nu)+\Delta r(\nu)$,

то порівнюючи з (43) бачимо, що від зміщення $z_{0}$ залежить тільки $\Delta r(\nu)$, причому $\Delta r\langle\langle R(\nu)$.

Здавалось би інтеграл $\int_{0}^{r_{m}(\nu)} d r r^{2}\left[\mathbf{K}_{0} \boldsymbol{\nabla} \Phi_{i}^{(0)}(r)\right]$ в

(45) можна розкласти у ряд по степенях $\Delta r_{m}(\nu)$ i отримати як лінійні, так і нелінійні по $z_{0}$ члени. Проте тут є один делікатний момент. Щоб отримати нелінійні по $z_{0}$ члени, виникає необхідність диференціювати підінтегральний вираз в (45), тобто функцію

$w(r)=r^{2}\left[\mathbf{K}_{0} \boldsymbol{\nabla} \Phi_{i}^{(0)}\right]$

\section{6}

Але як видно, наприклад, із точного виразу для іонного електростатичного потенціалу для сферичної форми $(34)$, функція $\Psi^{(s)}(r)$ та $\dddot{1}$ перша похідна неперервні в точці $r=R$, тобто на поверхні. Проте, вже друга похідна $\Psi^{(s)}(r)$ зазнає у точці $r=R$ розрив. Тому розкласти згаданий вище інтеграл у ряд Тейлора на поверхні не можна. Але зліва і справа від поверхні іонний електростатичний потенціал задано плавними функціями. Тому зробимо таким чином. Розіб'ємо в (45) інтеграл по $\nu$ на області, в яких $r_{m}(\nu)$ лежить тільки зліва або тільки справа від поверхні наночастинки. Тоді і зліва, і справа від поверхні виникають законні підстави для розкладання функції (47) у ряд Тейлора. Щоб був зрозумілий наш підхід, пояснимо його суть на прикладі сферичної форми, для якої результати вже відомі в $[1,2]$. Отже, інтеграл, який входить у (45) розпишемо так:

$$
\begin{aligned}
& \int_{0}^{\pi} d v \sin v \int_{0}^{r_{m}(v)} d r r^{2}\left[\mathbf{K}_{0} \boldsymbol{\nabla} \Phi_{i}^{(0)}\right]= \\
& =\int_{0}^{\pi / 2} d v \sin v \int_{0}^{r_{m}(v)} d r w(r)+\int_{\pi / 2}^{\pi} d v \sin v \int_{0}^{r_{m}(v)} d r w(r) .
\end{aligned}
$$

У першому інтегралі, як видно із $(44), r_{m}(\nu) \geq R$, а у другому $r_{m}(\nu) \leq R$ (наведені нерівності дещо порушуються при $v \approx \pi / 2$, але ця область не дає помітного внеску в інтеграл).

У випадку сферичної форми згідно з (34) і (37) ма€MO

$w(r)=-\rho_{i} V \cos \nu, \quad$ при $r \geq R$,

$w(r)=-\rho_{i} V \cos \nu\left(\frac{r}{R}\right)^{3}, \quad$ при $r \leq R$.

Підставимо тепер у перший і другий інтеграл у правій частині (48), відповідно, функції (49) і (50). Далі візьмемо до уваги, що $\mathbf{r}_{m}(\nu)=R+\Delta r(\nu)(\Delta r(\nu) \ll R)$ та використаємо розкладання

$$
\begin{aligned}
& \int_{0}^{r_{m}(\nu)} d r r^{2}\left[\mathbf{K}_{0} \nabla \Phi_{i}^{(0)}(r)\right]= \\
= & \int_{0}^{R+\Delta r(v)} d r w(r)=\int_{0}^{R} d r w(r)+w(r) \Delta r+\frac{1}{2}\left(\frac{d w}{d r}\right)_{R}(\Delta r)^{2}+
\end{aligned}
$$


$+\frac{1}{6}\left(\frac{d^{2} w}{d r^{2}}\right)_{R}(\Delta r)^{3}+\frac{1}{24}\left(\frac{d^{3} w}{d r^{3}}\right)_{R}(\Delta r)^{4}+\ldots$.

Щоб уникнути непорозуміння, ще раз відзначимо, що похідні в (51) беруться не на поверхні $(r=R)$, а в точці $r$ за умови, що $r$ прямує до поверхні зліва або справа. Зокрема, у даному конкретному випадку мова йде про розклад функції (50) і $r \rightarrow R$ при умові $r \leq R$.

Із виразів (43) і (44) бачимо, що $\Delta r$ це ряд по степенях $z_{0}$, тобто

$\Delta r=\Delta r_{1}+\Delta r_{2}+\Delta r_{3}+\Delta r_{4}$

де $\Delta r_{i} \sim z_{0}^{i}$. Зокрема у випадку сферичної форми згідно з (44) маємо

$\Delta r_{1}=z_{0} \cos v, \quad \Delta r_{2}=-\frac{z_{0}^{2}}{2 R} \sin ^{2} v$

$\Delta r_{3}=0, \quad \Delta r_{4}=-\frac{z_{0}^{4}}{8 R^{3}} \sin ^{4} v$

Зважаючи на (51) та (52), всі інтеграли в (45) беремо до кінця і отримуємо (при $z_{0} \geq 0$ ):

$\langle F\rangle=-e E_{L}(t) N_{e}-m_{e} N_{e} \omega_{p}^{2}\left\{z_{0}-\frac{9}{16} \frac{z_{0}^{2}}{R}+\frac{z_{0}^{4}}{32 R^{3}}\right\}$,

де $\omega_{p}^{2}=\frac{4 \pi e^{2} n_{0}}{3 m_{e}}-$ квадрат плазмової (дипольної) частоти. Отже, ми отримали результат робіт $[1,2]$. Тепер використаємо цей самий підхід для випадку сфероїдальної форми наночастинки.

\section{5. Нелінійні дипольні плазмові коливання асиметричної металевої наночастинки}

У випадку сфероїдальної форми наночастинки 3 огляду на (30) будемо мати

$$
\begin{aligned}
& \mathbf{K}_{0} \boldsymbol{\nabla} \Phi_{i}^{(0)}=\cos v \frac{\partial \Phi_{i}^{(0)}}{\partial r}-\frac{\sin v}{r} \frac{\partial \Phi_{i}^{(0)}}{\partial v}= \\
& =\cos v\left\{\frac{\partial \Psi(0)}{\partial r}+\frac{\partial \Psi(2)}{\partial r} P_{2}(\cos v)+\right. \\
& \left.+\frac{2}{r} \Psi(2)\left[1-P_{2}(\cos v)\right]+\ldots\right\} .
\end{aligned}
$$

Далі будемо явно враховувати тільки $\Psi(0)$, хоча необхідні обчислення було проведено також з урахуванням внеску функції $\Psi(2)$, вираз якої наведено в додатку. Як показали оцінки, врахування внеску $\Psi(2)$ дещо уточнює коефіцієнти при степенях $z_{0}$, але не змінюе основних висновків. Отже, згідно з (43) маєMO

$r_{m}(0)=R_{\|}+z_{0}>R_{\perp} ; \quad r_{m}(\pi)=R_{\|}-z_{0}<R_{\|}$.

3 огляду на ці нерівності знову інтеграл по кутах $v$ розіб'ємо відповідно до (48). У перший інтеграл правої частини (48), в якому згідно з (56) $r_{m}(0)>R_{\|}$, підставимо функцію (33). Відповідно до (47 і (30) будемо мати

$w(r)=\cos v r^{2} \frac{\partial \Psi(0)}{\partial r}=-\frac{4 \pi}{3} \rho_{i} R_{\perp}^{2} R_{\|} \cos v$.

$\mathrm{У}$ другому інтегралі (48), для якого $r_{m}(\pi)<R_{\|}$, будемо допускати також, що $r_{m}(v)>R_{\perp}$ і використовувати функцію (32). У цьому випадку будемо мати

$w(r)=\frac{4 \pi}{3} \rho_{i}\left\{\frac{1}{e_{p}}\left(r^{2}-R_{\perp}^{2}\right)^{3 / 2}-r^{3}\right\} \cos v$.

Зауважимо, що припущення $r_{m}(v)>R_{\perp}$ означає також, що

$r(\pi)=R_{\|}-z_{0}>R_{\perp}$ чи $z_{0}<R_{\|}-R_{\perp}$.

До тих пір, поки ми не робили припущення (59) як вирази для іонного електростатичного потенціалу, так і границі інтегрування (44) при $e_{p} \rightarrow 0$ переходили у відповідні результати для сферичної форми частинки. 3 прийняттям нерівності (59) подібний граничний перехід стає неможливим, оскільки при будь-якому малому, але фіксованому значенні $z_{0}$ при $e_{p} \rightarrow 0$ нерівність (59) перестає виконуватись. У цьому випадку, щоб зробити законним граничний перехід $e_{p} \rightarrow 0$ замість функції (32) потрібно було би брати функцію (31). Отже, підстановка (57) і (58) в (48) дає

$$
\begin{aligned}
& \int_{0}^{\pi} d v \sin v \int_{0}^{r_{m}(v)} d r\left[\mathbf{K}_{0} \boldsymbol{\nabla} \Phi_{i}^{(0)}\right]= \\
& =-\frac{4 \pi}{3} R_{\perp}^{2} R_{\|} \rho_{i} \int_{0}^{\pi / 2} d v r_{m}(v) \sin v \cos v+ \\
& +\frac{4 \pi}{3} \rho_{i} \int_{\pi / 2}^{\pi} d v \sin v \cos v \int_{0}^{r_{m}(v)} d r\left\{\frac{1}{e_{p}}\left(r^{2}-R_{\perp}^{2}\right)^{3 / 2}-r^{3}\right\} .
\end{aligned}
$$


Щоб взяти другий інтеграл по $r$ в (60) використаємо подібно (51) малість величини $\Delta r$ і розкладемо цей інтеграл в ряд по $\Delta r$. Проте є певна різниця між випадками (51) і (60). У формулі (51) $r_{m}(v)=R+\Delta r(v)$, а в $(60)$ відповідно до $(43) r_{m}(v)=R(v)+\Delta r(v)$. Щоб уникнути зайвих ускладнень, ми будемо розкладати $\int_{0}^{r_{m}(v)} d r\{\ldots\}$ по $\Delta r$ не в точці $R(v)$, а в точці $R_{\| \cdot}$ Підставою для такого наближення є те, що по-перше, електростатичний потенціал визначається переважно розподілом зарядів біля вершини (полюса) еліпсоїда, тобто діапазоном кутів, в якому $R(v) \approx R_{\|}$, а подруге, у точці $r=R_{\|}$функція $\Psi(0)$, і $\dddot{1}$ похідна, як видно із (32), (33), неперервні подібно до сферичного випадку (де відомий точний розв'язок).

Якщо обмежитись кубічними по $\Delta r$ членами, то із (60) отримуємо

$$
\begin{aligned}
& \int_{0}^{\pi} d v \sin v \int_{0}^{r_{m}(v)} d r r^{2}\left[\mathbf{K}_{0} \nabla \Phi_{i}^{(0)}\right]= \\
& =-V \rho_{i} \int_{0}^{\pi / 2} d v \Delta r(v) \sin v \cos v- \\
& -V \rho_{i} \int_{\pi / 2}^{\pi} d v \sin v \cos v\left\{\Delta r(v)-\frac{(\Delta r(v))^{3}}{2 e_{p}^{2} R_{\|}^{2}}\right\}
\end{aligned}
$$

де $V=\frac{4 \pi}{3} R_{\perp}^{2} R_{\|}$- об'єм сфероїда.

Зауважимо, що завдяки нерівності (59) справедлива також, як неважко переконатись, нерівність

$e_{p} R_{\|}>z_{0}$

При виконанні нерівності (62) кубічний по $\Delta r$ член значно менший від лінійного, як і повинно бути при розкладанні у ряд по малому параметру. Оскільки вираз $\Delta r(v)$ сам є розкладом у ряд по $z_{0}$, то ми надалі будемо обмежуватись внеском членів не вище $z_{0}^{3}$, тобто будемо підставляти в (61):

$\Delta r(v) \approx \Delta r_{1}(v)+\Delta r_{2}(v)+\Delta r_{3}(v)$,

$(\Delta r(v))^{3} \approx\left(\Delta r_{1}(v)\right)^{3}$

Вигляд членів $\Delta r_{i}(v)$ для сфероїдальної форми стає ясним із виразу (43).
Взявши відповідні інтеграли в (61) і підставивши отриманий вираз в (45), будемо мати

$$
\langle F\rangle=-e E_{L}(t) N_{e}-m_{e} N_{e} \omega_{\|}^{2} z_{0}-m_{e} N_{e} \omega_{p L}^{2} \frac{\delta\left(e_{p}\right)}{R_{\|}^{2}} z_{0}^{3}
$$

$\omega_{\|}^{2}=L_{\|} \omega_{p L}^{2} \equiv \frac{1-e_{p}^{2}}{2 e_{p}^{3}}\left\{\ln \left(\frac{1+e_{p}}{1-e_{p}}\right)-2 e_{p}\right\} \omega_{p L}^{2}$,

де $\omega_{p L}=\left(\frac{4 \pi n_{0} e^{2}}{m_{e}}\right)^{1 / 2}-$ плазмова частота, $L_{\|}-$коефіцієнт деполяризації вздовж осі симетрії для випадку витягнутого сфероїда $\left(R_{\|}>R_{\perp}\right)$. Крім того, в (64) введено залежний тільки від ексцентриситету $\left(e_{p}\right)$ безрозмірний параметр $\delta\left(e_{p}\right)$, який має вигляд

$$
\begin{aligned}
& \delta\left(e_{p}\right)=\frac{4}{315} \frac{e_{p}^{2}}{1-e_{p}^{2}}-\frac{13}{12 e_{p}^{2}}+\frac{5}{2 e_{p}^{4}}- \\
& -\frac{5-6 e_{p}^{2}+e_{p}^{4}}{8 e_{p}^{5}} \ln \left(\frac{1+e_{p}}{1-e_{p}}\right)- \\
& -\frac{1-e_{p}^{2}}{16 e_{p}^{4}}\left\{\frac{5}{2}-\frac{3}{2 e_{p}^{2}}+\frac{3\left(1-e_{p}^{2}\right)^{2}}{4 e_{p}^{3}} \ln \left(\frac{1+e_{p}}{1-e_{p}}\right)\right\} .
\end{aligned}
$$

Порівнюючи вирази для електростатичної сили у випадку сферичної форми (формула (54)) і у випадку еліпсоїдальної форми (формула (64)) бачимо, що в асиметричної частинки зникає квадратична нелінійність, однак з'являється кубічна нелінійність. Ще раз нагадаємо, що вираз (66) отриманий в допущенні, що $0<e_{p}<1$, і тому граничний перехід $e_{p} \rightarrow 0$ чи $e_{p} \rightarrow 1$ не може бути виправданим. Для орієнтації про порядок величини $\delta\left(e_{p}\right)$ приводимо значення

$\delta\left(\frac{1}{2}\right)=-\frac{1}{7}, \quad \delta\left(\frac{1}{5}\right)=-1$.

Рівняння руху (5) для нашого випадку, коли коливання відбуваються вздовж осі симетріі $\left(\mathbf{u}=\mathbf{z}_{0}\right)$, з урахуванням виразу (64), набуває вигляду

$\ddot{z}_{0}+\omega_{\|}^{2} z_{0}+\omega_{p L}^{2} \frac{\delta\left(e_{p}\right)}{R_{\|}^{2}} z_{0}^{3}=-\frac{e E(t)}{m_{e}}$.

Частота $\omega_{\|}$відповідає частоті дипольного плазмона при коливанні диполя вздовж осі симетрії сфероїда. Якщо задати

$E_{L}(t)=E_{0} \cos \omega t$

\section{8}


та вважати нелінійність слабкою, то рівняння (67) можна розв'язати методом послідовних наближень і отримати

$z_{0} \approx \frac{e E_{0}}{m} \frac{\cos \omega t}{\omega_{\|}^{2}-\omega^{2}}+\frac{\omega_{p L}^{2} \delta\left(e_{p}\right)}{4 R_{\|}^{2}} \frac{\left(e E_{0} m_{e}\right)^{3}}{\left(\omega_{\|}^{2}-\omega^{2}\right)^{3}} \times$

$\times\left\{\frac{3 \cos \omega t}{\omega_{\|}^{1}-\omega^{2}}+\frac{\cos 3 \omega t}{\omega_{\|}^{2}-(3 \omega)^{2}}\right\}$.

Оскільки ми в (67) не врахували дисипації, то розв'язок при $\omega \rightarrow \omega_{\|}$має сингулярність. Якщо, як це прийнято, додатковим членом $2 \gamma z_{0}$ у лівій частині (67) врахувати згасання коливань, то сингулярність у розв'язку зникне. Зокрема в лінійному розв'язку в цьому випадку замість $\frac{e E_{0}}{m} \frac{\cos \omega t}{\omega_{\|}^{2}-\omega^{2}}$ будемо мати $\frac{e E_{0}}{m} \frac{\cos \left(\omega t-\varphi_{0}\right)}{\left\{\left(\omega_{\|}^{2}-\omega^{2}\right)^{2}+4 \gamma^{2}\right\}^{1 / 2}}$, де $\varphi_{0}-$ фаза. Аналогічні зміни потрібно зробити і в нелінійних членах. Зауважимо, що рівняння $(67)$ з виглядом $E_{L}(t)$, заданим у формі (68), відповідає так званому рівнянню Дуффінга. Аналіз його розв'язків можна знайти, наприклад, у роботі [5].

Тепер, коли маємо явний вигляд зміщення електронного центра мас $z_{0}$ як функції лазерного поля $E_{0}$ у формі (69), можемо записати дипольний момент сфероїдальної металевої наночастинки. У випадку поляризації лазерного поля вздовж осі симетрії сфероїда дипольний момент має ту саму орієнтацію і дорівнюе

$d=V e n_{0} z_{0}=d_{1}+d_{2}$,

де $d_{1}$ - лінійна складова диполя, яка відповідно до (69) дорівнює:

$d_{1}=\frac{V}{4 \pi} \frac{\omega_{p L}^{2}}{\omega_{\|}^{2}-\omega^{2}} E_{0} \cos \omega t$

Аналогічно кубічна складова диполя $d_{3}$ може бути записана у вигляді

$$
\begin{aligned}
& d_{3}=\frac{V \delta\left(e_{p}\right)}{16 \pi R_{\|}^{2}} \frac{(e m)^{2} \omega_{p L}^{3}}{\left(\omega_{\|}^{2}-\omega^{2}\right)^{3}} E_{0}^{3} \times \\
& \times\left\{\frac{3 \cos \omega t}{\omega_{\|}^{2}-\omega^{2}}+\frac{\cos 3 \omega t}{\omega_{\|}^{2}-(3 \omega)^{2}}\right\} .
\end{aligned}
$$

На закінчення зауважимо таке. Якщо ввести безрозмірне зміщення $\alpha \equiv z_{0} / R_{\|}$, то рівняння (67) набуває вигляду

$\ddot{\alpha}+\omega_{\|}^{2} \alpha+\omega_{p L}^{2} \delta\left(e_{p}\right) \alpha^{3}=\frac{e E(t)}{m R_{\|}}$.

Як бачимо, величина нелінійності визначається безрозмірним параметром $\delta\left(e_{p}\right)$, аналітичний вигляд якого як функції ексцентриситету $e_{p}$ заданий формулою (66). Крім того, конкретні значення $\delta\left(e_{p}\right)$ при $e_{p}=1 / 2, e_{p}=1 / 5$ наведено вище. Це дозволяє оцінювати величину нелінійності.

Варто також відзначити, що розглянута вище нелінійність зумовлена електричною складовою лазерної хвилі. За певних умов (розмірах частинок, частотах) нелінійність може бути індукована магнітною складовою. Зокрема ефект генерації другої гармоніки в сферичних металевих частинках під дією магнітної складової лазерної хвилі розглянуто в роботі [6].

\section{6. Висновки}

Показано, що в металевій наночастинці сфероїдальної форми лазерним полем, орієнтованим вздовж осі симетрії частинки, генерується кубічна нелінійність, яка була відсутня у випадку сферичної форми. Зате зникає квадратична нелінійність, наявна у сферичному випадку.

Отримано наближений аналітичний вираз для дипольного моменту сфероїдальної металевої наночастинки з точністю до кубічних по полю членів.

\section{ДОДАТОК}

$\Psi(2)=2 \pi \rho_{i} r^{2}\left\{\frac{1}{3}-\frac{e_{p}^{2}-1}{e_{p}^{2}}+\frac{e_{p}^{2}-1}{2 e_{p}^{3}} \ln \left(\frac{1+e_{p}}{1-e_{p}}\right)\right\}$,

при $\quad r \leq R_{\perp}$.

$\Psi(2)=2 \pi \rho_{i} r^{2}\left\{\begin{array}{l}\frac{1}{3}\left(1-\cos ^{3} \theta_{1}\right)+\frac{1}{5}\left(1-\cos ^{2} \theta_{1}\right) \cos \theta_{1}- \\ -\frac{e_{p}^{2}-1}{e_{p}^{2}}\left(1-\cos \theta_{1}\right)- \\ -\frac{e_{p}^{2}-1}{2 e_{p}^{3}} \ln \left[\frac{1+e_{p} \cos \theta_{1}}{1-e_{p} \cos \theta_{1}} \frac{1-e_{p}}{1+e_{p}}\right]+ \\ +\frac{1}{15}\left[\left(\frac{R_{\perp}}{r}\right)^{2}\left(3 \cos ^{2} \theta_{1}-1\right)-2\left(\frac{R_{\perp}}{r}\right)^{4}\right] \cos \theta_{1} \\ \text { при } \quad R_{\perp} \leq r \leq R_{\|}\end{array}\right\}$,

$\Psi(2)=\frac{4 \pi}{15} \rho_{i} R_{\perp}^{2} e_{p}^{2}\left(\frac{R_{\|}}{r}\right)^{3}, \quad$ при $r \geq R_{\|}$.

1. P.B. Parks, T.F. Cowan, R.B. Stephens, and E.M. Campbell, Phys. Rev. A 63, 063203 (2001).

2. S.V. Fomichev, S.V. Popruzhenko, D.F. Zaretsky, and W. Becker, J. Phys. B 36, 3817 (2003). 
3. L.G. Gerchikov, C. Guet, and A.N. Ipatov, Phys. Rev. A 66, 053202 (2002).

4. Г. Арфкен, Математические методъ в физике (Атомиздат, Москва, 1970).

5. Дж. Стокер, Нелинейные колебания в механических и электрических системах (Изд. Иностранной Литературы, Москва, 1953).

6. Y. Zeng, W. Hoyer, J. Lin, S.W. Koch, and J.V. Moloney, arXiv: $0807.3575 \mathrm{v} 2$.

НЕЛИНЕЙНЫЕ ПЛАЗМОВЫЕ ДИПОЛЬНЫЕ

КОЛЕБАНИЯ В СФЕРОИДАЛЬНЫХ

МЕТАЛЛИЧЕСКИХ НАНОЧАСТИЦАХ

П.М. Томчук, Д.В. Бутенко

$\mathrm{P}$ е $з$ ю м е

В работе развита теория нелинейных дипольных плазмовых колебаний в металлической наночастице сфероидальной формы, которые генерируются полем лазерной волны. Для слу- чая лазерного поля, ориентированного вдоль оси вращения сфероида, получены приближенные аналитические выражения для дипольного момента наночастицы (с точностью до кубической составляющей).

\section{NONLINEAR PLASMA DIPOLE OSCILLATIONS}

IN SPHEROIDAL METAL NANOPARTICLES

\section{P.M. Tomchuk, D.V. Butenko}

Institute of Physics, Nat. Acad. of Sci. of Ukraine,

(46, Prosp. Nauky, Kyiv 03680, Ukraine;

e-mail: ptomchuk@iop.kiev.ua)

$\mathrm{S}$ u m m a r y

The theory of nonlinear dipole plasma oscillations generated in a metal spheroidal nanoparticle by a laser-wave field has been developed. Approximate (to an accuracy of the cubic term) analytical expressions for the nanoparticle dipole moment have been obtained in the case of the laser field oriented along the spheroid rotation axis. 